\title{
Single Perceptron Model for Smart Beam Forming in Array Antennas
}

\author{
K. S. Senthilkumar ${ }^{1}$, K. Pirapaharan ${ }^{2}$, P. R. P. Hoole ${ }^{3}$, H. R. H. Hoole ${ }^{4}$ \\ ${ }^{1}$ Department of Mathamatics and Computer Science, Papua New Guinea University of Technology, PNG \\ ${ }^{2}$ Department of Electrical and Communications Engineering, Papua New Guinea University of Technology, PNG \\ ${ }^{3}$ Department of Electrical and Electronic Engineering, Faculty of Engineering, Universiti Malaysia Sarawak, Malaysia \\ ${ }^{4}$ Department of Electrical and Computer Engineering, Michigan State University, USA
}

\begin{tabular}{l}
\hline \hline Article Info \\
\hline Article history: \\
Received Apr 4, 2016 \\
Revised Aug 7, 2016 \\
Accepted Aug 28, 2016 \\
\hline
\end{tabular}

Keyword:

Adaptive array

Adaptive beamforming

Artificial neural network

Smart antenna

\begin{abstract}
In this paper, a single neuron neural network beamformer is proposed. A perceptron model is designed to optimize the complex weights of a dipole array antenna to steer the beam to desired directions. The objective is to reduce the complexity by using a single neuron neural network and utilize it for adaptive beamforming in array antennas. The selection of nonlinear activation function plays the pivotal role in optimization depends on whether the weights are real or complex. We have appropriately proposed two types of activation functions for respective real and complex weight values. The optimized radiation patterns obtained from the single neuron neural network are compared with the respective optimized radiation patterns from the traditional Least Mean Square (LMS) method. Matlab is used to optimize the weights in neural network and LMS method as well as display the radiation patterns.
\end{abstract}

Copyright $\mathbb{C} 2016$ Institute of Advanced Engineering and Science. All rights reserved.

\section{Corresponding Author:}

K. S. Senthilkumar,

Department of Mathamatics and Computer Science,

Papua New Guinea University of Technology,

Lae 411, PNG.

Email: ksskumar16@gmail.com

\section{INTRODUCTION}

Due to its broad range of applications, adaptive array antenna is most popular in the present world. Present world applications require much faster beam steering that cannot be achieved using a mechanical systems. Hence it is required to use more consistent and much faster electronic beam steering techniques such as adaptive arrays. However, the beamforming withal most identical elements result lack of flexibility. On the other hand, adaptive beamforming methods by means of weight optimization are capable of managing the complexities of distinct elements. The adaptive array can detect, track and allocate narrow beams in the direction of the desired users while nulling unwanted sources of interferences. There are well known traditional techniques for adaptive beamforming in array antenna.

Soft computing techniques namely Artificial Neural Networks (ANN), fuzzy logics, Genetic Algorithms (GAs), provide low cost solutions and robustness to different complex real world problems. ANN is a powerful information processing paradigm that tries to simulate the structure and functionalities of the biological nervous systems. The ANN is used to deal with many applications, and they have proved their effectiveness in several research areas such as image recognition, speech recognition, signal analysis, process control, and robotics. The true power of neural networks lies in their ability to represent both linear and nonlinear relationships. ANN, like human learning, learns by example. Training a neural network is, in most cases, an exercise in numerical optimization of a usually nonlinear function. Basic building block of every artificial neural network is an artificial neuron or perceptron that is a simple mathematical model. 
Many researchers have been using Neural Network methods in antenna array signal processing. Neural networks are used in adaptive antenna signal processing [1]-[2] because of their general purpose nature, fast convergent rate and large scale integration implementations. The goal of neural network training is to minimize the difference between output data and the target data. Neural network techniques have advantages such as nonlinear property, adaptive learning capability, and fast convergence rates. These characteristic advantages of the neural network encourage us to use in adaptive beamforming. Identifying the inherent gains of neural networks, a number of literatures are available on neural network based model to calculate the weights of an adaptive array antenna [3]-[6].

A complex multilayered Radial Basis Function Neural Network (RBFNN) is proposed to successfully examine the adaptive beamforming of nearfield Ultra Wide band (UWB) array [7]. The authors claimed that RBFNN method is derived from regular theory, has the optimal approximation ability to complicated functions and has a faster learning speed compared to global methods, such as the MLP with BPrule. Where they uses a neural network with number of nodes in input layer, hidden layer and output layer and use genetic algorithm (GA)to determine the number of hidden neurons. Mohamad [8] has also carried out a comparative performance analysis on stochastic and GA algorithms on smart antennas for dynamically changing environment.

A neural-fuzzy based composite technique is advocated to design the adaptive beamformer [9]. Where the authors use feed forward neural network for training the weight vectors for different angle of incidence of the signal to arrive at the initial estimate of the weights and fine tunes the weights till the maximum signal output power is reached. The weight is calculated in each iteration till the error is minimized and convergence of weight is achieved. They have shown that the convergence time in their algorithm is faster than the LMS algorithms using simulation.

However, we propose a single neuron weight optimization model (SNWOM) for adaptive beamforming in smart antennas by carefully selecting the appropriate activation functions to match with the desired radiation pattern and the dipole location.

\section{SINGLE NEURON WEIGHT OPTIMIZATION MODEL (SNWOM)}

Here we briefly describe the single neuron modal to optimize the weights which will be used in adaptive beamforming. In the perceptron model as shown in Figure 1, a single neuron with a linear weighted net function and a threshold activation function also known as transfer function is employed. The model has three parts and at the first part inputs $\left(x_{1}, x_{2} \ldots, x_{n}\right)$ are multiplied with individual weights $\left(w_{1}, w_{2} \ldots w_{n}\right)$. In the second part of simple perceptron is the net function that sums all weighted inputs and bias as:

$$
z=b+\sum_{k=1}^{n} w_{k} x_{k}
$$

In the final part of simple perceptron the sum of previously weighted inputs and bias is passing through a transfer function to get the output. In case of linear activation function artificial neuron is doing simple linear transformation over the sum of weighted inputs and bias $b$. There is no single best method for nonlinear optimization and is based on the characteristics of the problem to be solved.

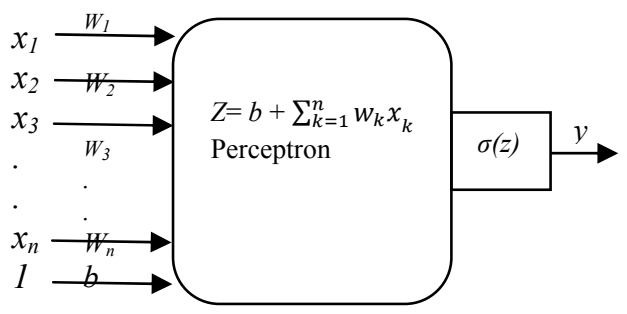

Figure 1. Perceptron model for weight optimization

We simplify the calculation complexity to reduce the processing delay. Hence we have used single neuran for this problem and a nonlinear activation function $\sigma$ to find out the output $y$ as when the weights are real value. 


$$
y=\sigma(z)=\frac{1}{1+e^{-z}}
$$

Many neural network architectures generally operate on real data. But there are many applications where consideration of complex inputs is quite desirable. Trainingthe neural networks using complex inputs was done using techniques like the back-propagation, Hopfield model and perceptron learning rules. Their performances were tested using the pattern classification [10], signal processing [11] and time series experiments and its generalization capability was found to be satisfactory.

In complex-valued neural network, inputs, output, threshold, and weights are complex values and selecting activation function is a challenging pare. Because of the neural network's outstanding capability of fitting on non-linear models many researches have been done in the recent past [12]-[13]. Hamid et al [14] have studied new types of complex-valued sigmoid activation function for multi-layered neural network. Their simulation results proved that their proposed network reduced $54 \%$ of testing time compared to neural network uses normal sigmoid activation function as given below.

$$
y=\sigma(z)=\frac{\tanh (\operatorname{Re}(z))}{1-\left(\operatorname{Re}(z)-C_{r}\right) e^{-\operatorname{Re}(z)}}+j \frac{\tanh (\operatorname{Im}(z))}{1-\left(\operatorname{Im}(z)-C_{i}\right) e^{-\operatorname{Im}(z)}}
$$

The coefficients $C_{r}$ and $C_{i}$ can be adjustable to achieve the fast optimization with high accuracy. In order to train the weights to meet the desired output of $y_{0}$, the deviation $\Delta$ is obtained and the weights are iterated until it reach the trained means error TMR is below the predefined value. Where the deviation, and trained means error as below.

$$
\begin{aligned}
& \Delta=\left|y_{0}-y\right| \\
& T M R=\frac{\Delta}{y_{0}} * 100
\end{aligned}
$$

Also the weights are adjusted in every iteration using the deviation and the selected learning rate also known as coefficient $k_{0}$ as given below:

$$
w_{i}=w_{i}+k_{0} \times \Delta \times x_{i}
$$

The iteration is allowed either it reaches the $T M R$ below the predefined value $T M R_{m}$ or the defined maximum number $N$ of iterations. The SNWOM weight optimization flowchart is given in Figure 2.

\section{ADAPTIVE ARRAY MODEL}

In adaptive array design, the placement of dipoles can be any manner since the current amplitude and the phase could be adjustable to get the desired radiation patterns. However, analytically we can show that for any arbitrary set of dipoles arranged in a straight line would produce a radiation pattern that is symmetrical on both side of the plane where the dipoles are placed. As a result, the placement of dipole must be chosen based on the desired radiation patterns. If the set of desired radiation patterns are symmetrical on a common axis then the dipoles can be placed in that common axis where all the current components will in phase with different set of amplitudes with respective radiation patterns. Alternatively, when the set of desired radiation patterns are unsymmetrical on a common axis, the placement of diploes will not be in a common axis while the current components will be in different phases and amplitudes. Consequently, the in phase and the different phase current components will result real and complex optimized weight values, respectively. Therefore, we have proposed two types of activation functions for optimizing real and complex weights. Accordingly, we model a general setup as shown in Figure 3 where $n$ numbers of dipoles are placed arbitrarily.

For the arbitrary placement of dipoles, the currents will be different in phase amplitude and it can be represented by complex current phasors. The respective complex current phasors of the dipoles are taken as $I_{1}, I_{2}$, and $I_{n}$. Hence the electric field (far-field) at the observation point $\mathrm{P}$ could be given as: 


$$
E=A_{0} I_{1} e^{-j \beta r_{1}}+A_{0} I_{2} e^{-j \beta r_{2}}+\ldots+A_{0} I_{n} e^{-j \beta r_{n}}
$$

where $A_{0}$ and $\beta$ are a constant and the phase constant, respectively.

Substituting for $r_{1}, r_{2}$, and $r_{n}$ in terms of the distance from origin, equation (7) can be simplified to:

$$
E=w_{1} e^{j \beta\left(x_{1} \cos \varphi+y_{1} \sin \varphi\right)}+w_{2} e^{j \beta\left(x_{2} \cos \varphi+y_{2} \sin \varphi\right)}+\ldots+w_{n} e^{j \beta\left(x_{n} \cos \varphi+y_{n} \sin \varphi\right)}
$$

where $w_{1}, w_{2}$, and $w_{\mathrm{n}}$ are the complex weights and in proportional to the complex current phasors $I_{1}, I_{2}$, and $I_{n}$, respectively. To achieve the objective of forming a resultant single beam, the value of the complex weights $w_{1}, w_{2}$, and $w_{\mathrm{n}}$ needs to be optimized such that the resultant field must matched to a desired single beam function $f(\varphi)$. Thus equation (8) can be written as,

$$
w_{1} e^{j \beta\left(x_{1} \cos \varphi+y_{1} \sin \varphi\right)}+w_{2} e^{j \beta\left(x_{2} \cos \varphi+y_{2} \sin \varphi\right)}+\ldots+w_{n} e^{j \beta\left(x_{n} \cos \varphi+y_{n} \sin \varphi\right)}=f(\varphi)
$$

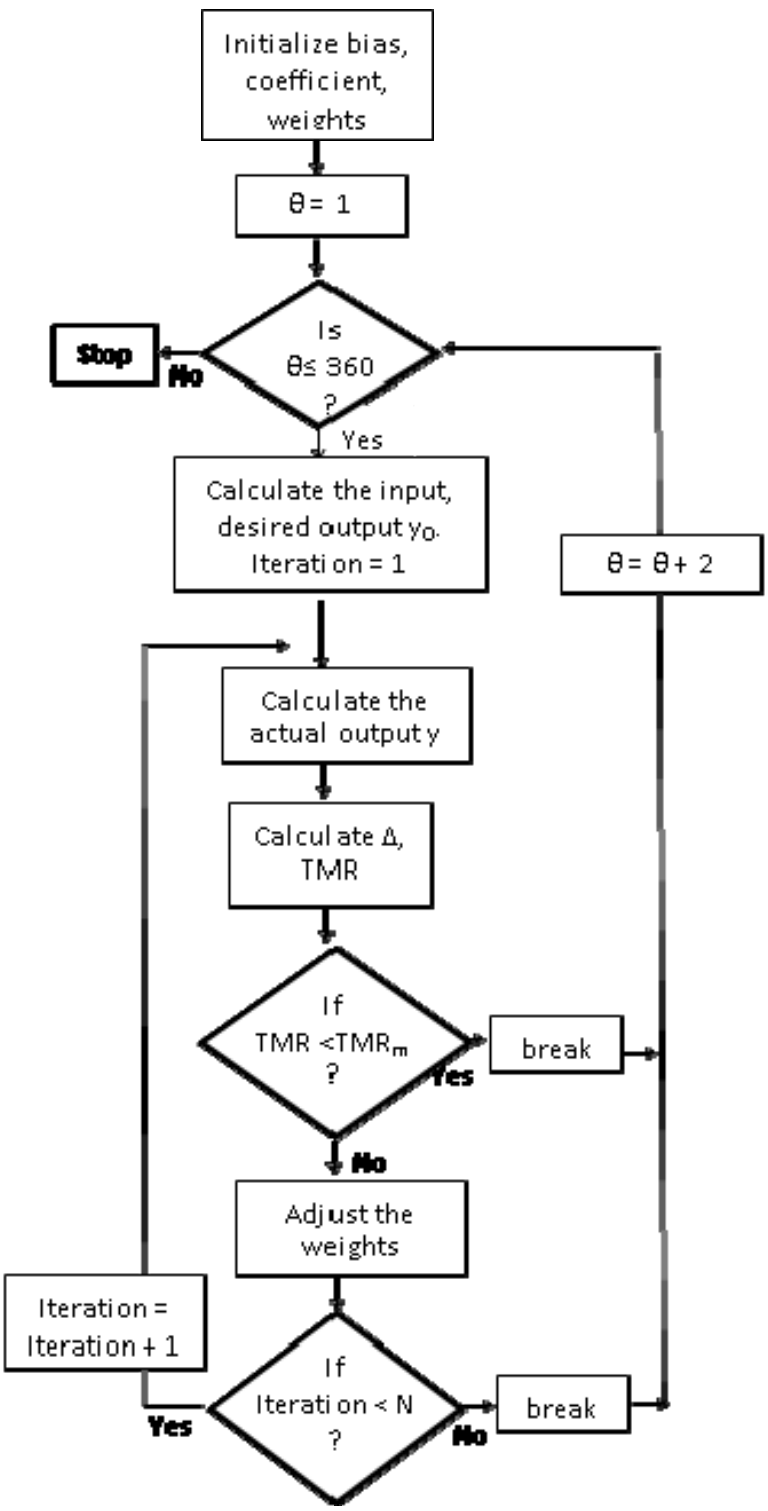

Figure 2. The SNWOM weight optimization 


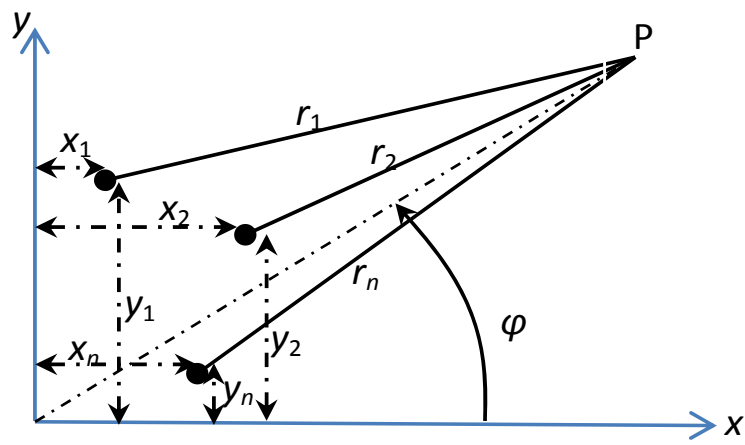

Figure 3. Schematic Diagram of Dipole Placement

\section{RESULTS AND DISCUSSION}

\subsection{Real Value Weights}

The dipoles are placed on a straight line by fixing the desired beam function $f(\phi)$ as $\cos 2 \phi$ and taking the distance between two elements as half wavelength, we have optimized the weights for five and seven elements to find the actual output using the above SNWOM model with initial weights, bias and learning rate also known as coefficient. For training, we have used different angles $\phi$ in the range of $0^{0}$ to $360^{\circ}$. During the testing process we have used different angles $\phi$ in the range of $0^{\circ}$ to $360^{\circ}$. Having obtained the optimized weights after convergence, we have drawn the radiation patterns using optimized weights and compared with the radiation patterns of the desired beam for five elements array as shown in the Figure 4. The Perceptron generated antenna beam is seen to closely match with the desired beam with which it is to be optimized. The beam is seen to give the maximum radiation in the desired direction and null points that match the desired beams null points. The beamwidth is wider, thus leading to some interference when receiving, as well as a measure of power wastage when transmitting. It is seen that there is maximum radiation over a wider area than what is required by the desired beam. However, as shown in Figure 5, this is rectified by increasing the number of elements from five to seven, thus getting greater accuracy at a cost. It should be remembered that whereas most multilayer neural network beam formers as well as the optimization procedures that use algorithms such as the least mean square methods (LMS) requires heavy computational time and memory to store multilayer weights, for instance, the Perceptron requires little memory and gives fast convergence when training and rapid generation of the beam when in operation on line. The beams of Figure 4 to 7 are beams required to communicate from the junction of underground tunnels in mines, as well as along the streets with vehicles from a base station beam at a junction.

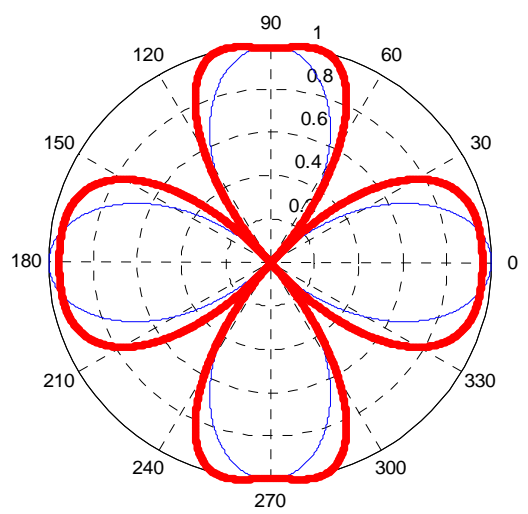

— Desired Beam $\quad * * * * * * *$ Optimized Beam

Figure 4. Comparison of Radiation pattern between optimized beam and desired beam obtained by SNWOM when the number of adaptive array elements is five

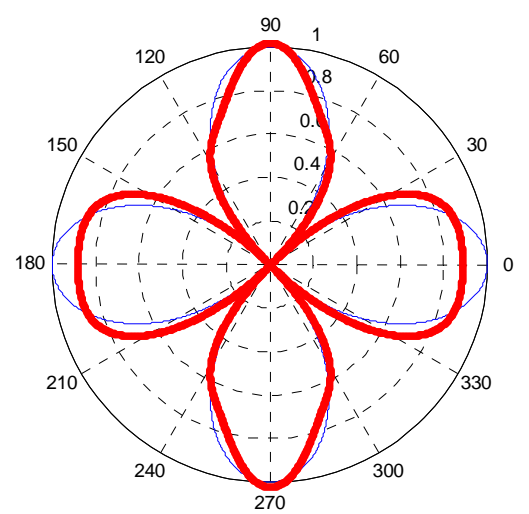

— Desired Beam $\quad * * * * * *$ Optimized Beam

Figure 5. Comparison of Radiation pattern between optimized beam and desired beam obtained by SNWOM when the number of adaptive array elements is seven 
Similarly, we have optimized the weights using stated SNWOM. The optimized results are shown for seven elements as in Figure 5. As we have expected, with increased number of elements, the adaptive array beamforming is very much close to the desired beam. However the amplitudes in the $0^{\circ}$ and $180^{\circ}$ are better in five elements array than seven elements array that due to the characterstic of the desired beam selected.

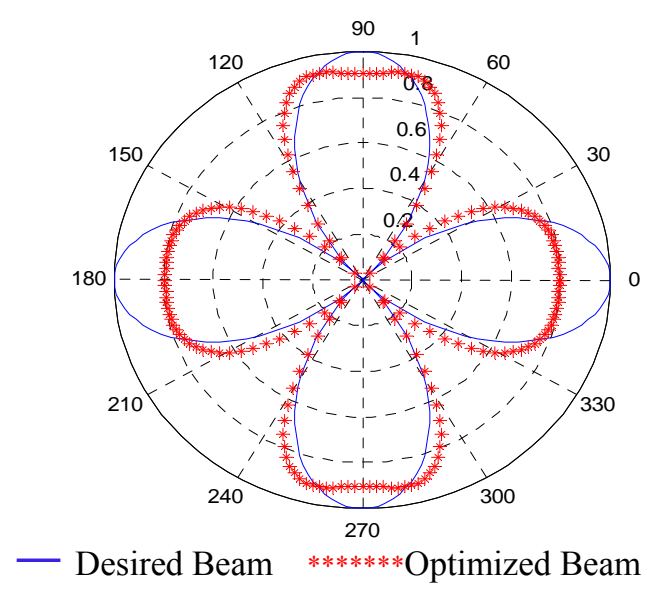

Figure 6. Comparison of Radiation pattern between optimized beam and desired beam when the number of adaptive array elements is five

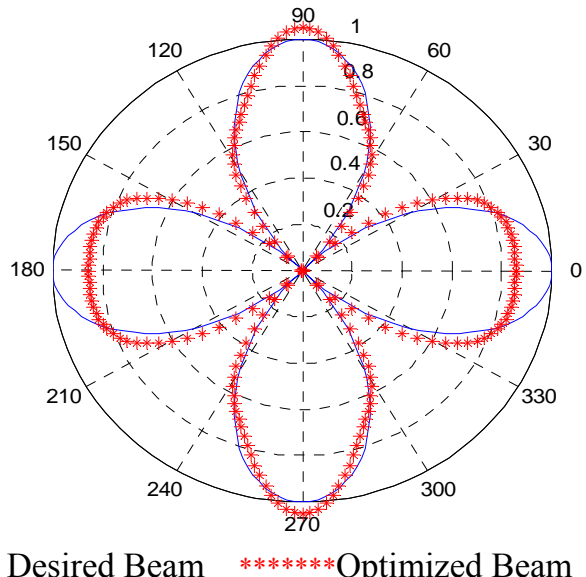

Figure 7. Comparison of Radiation pattern between optimized beam and desired beam when the number of adaptive array elements is seven

In order to have the comparison between accuracy of weights optimized from SNWOM method with the weights optimized from traditional LMS method, the weights are calculated for five elements and seven elements array antenna using LMS optimization. The radiation patterns for five and seven elements optimized from LMS methods are shown in Figure 6 and Figure 7, respectively.

Comparison of Figure 4 and Figure 6 displays that the results obtained from SNWOM has better match than LMS method though significant difference cannot be observed between Figure 5 and Figure 7.

In order to further test the precision of the SNWOM method with variety of desired function, we select a desired function as [15]:

$$
f(\phi)=\frac{1}{9}|3+4 \cos (\pi \cos \phi)+2 \cos (2 \pi \cos \phi)|
$$

We optimize weights by taking the distance between two elements as half wavelength for five and seven elements using SNWOM. The optimized radiation patterns are compared with the desired radiation patterns in Figure 8 and Figure 9 for five and seven elements, respectively. We observe the close match between desired and optimized radiation patterns. It is evident from the results that the desired narrow beam could not be achieved using 5 elements model while it is feasible with 7 element model. Therefore, it can be recognized that the narrow desired beam require more number of dipole elements. The side lobes of this broadside antenna are relatively small, as seen in Figure 8. Where this linear array antenna need to be used as a single beam antenna, a reflector may be used to flip over the unwanted of the two main beams. The Perceptron beamforming method proposed may work with any chip-based MIMO techniques, including transmit beam forming, spatial multiplexing, space-time block coding and cyclic delay diversity. 


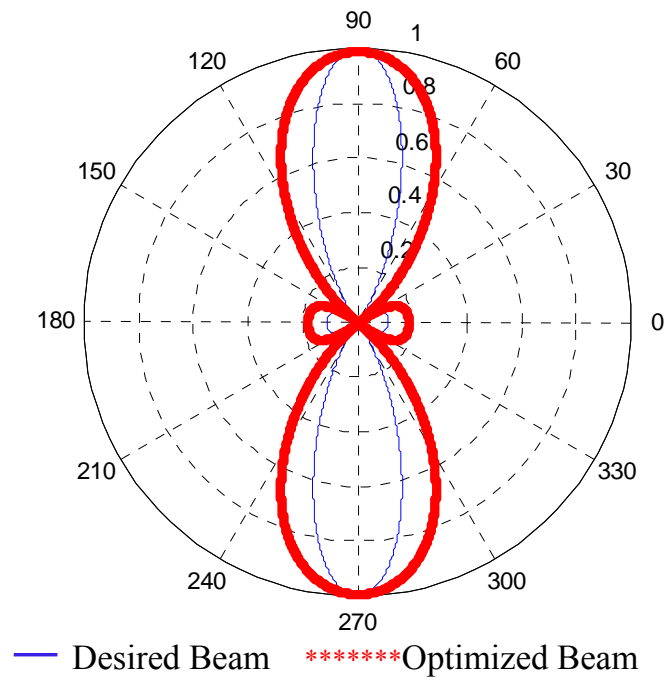

Figure 8. Comparison of Radiation pattern between optimized beam and desired beam obtained by SNWOM when the number of adaptive array elements is five

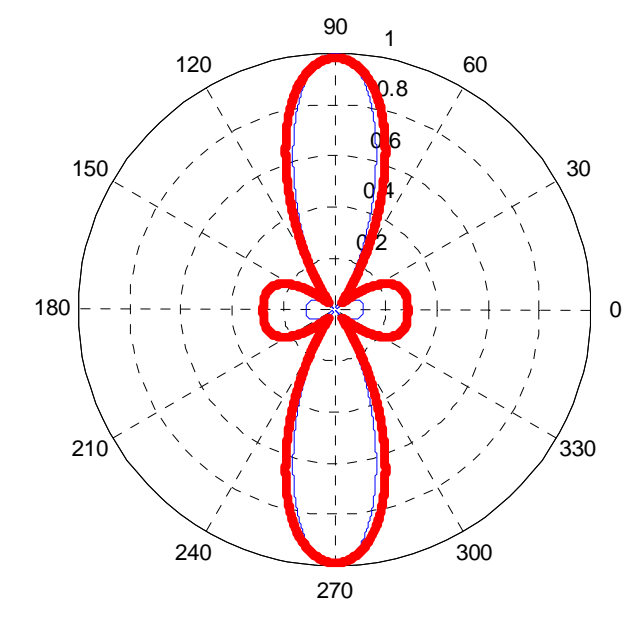

- Desired Beam $\quad * * * * * * *$ Optimized Beam

Figure 9. Comparison of Radiation pattern between optimized beam and desired beam obtained by SNWOM when the number of adaptive array elements is seven

Since only a single Perceptron is used for beamforming, the technique is fast, and could provide the best set of antenna patterns within milliseconds. Even though, the precision of the SNWOM is depending on the dipole placement and the characteristics of the desired beam selected, it is a fast, efficient and simple method for the weight optimization compared to the previously proposed neural network based adaptive beamforming methods.

\subsection{Complex Value Weights}

When the desired radiation patterns are unsymmetrical on common axis then the placement of the dipoles cannot be in common axis as discussed earlier. Bodhe et al [16] have proposed a rectangular array structure to provide solution for such condition. However, it is possible to construct an array with minimum three elements that cannot be in common axis which will lead to complex weight values. Therefore we have considered the minimum three along with four and six element arrays to compare the accuracy of beamforming as shown in Figure 10. The desired function is selected as $f(\varphi)=\operatorname{sinc}\left(\varphi-\varphi_{0}\right)$ to form a single beam, where $\varphi_{0}$ is the desired angle.

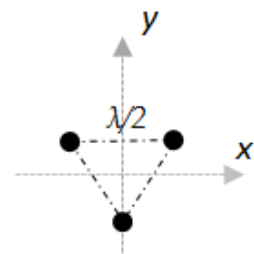

(a)

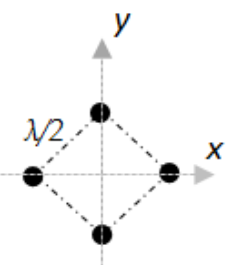

(b)

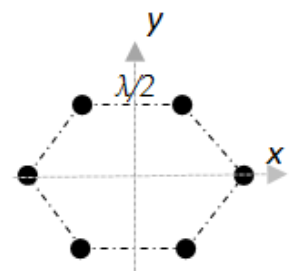

(c)

Figure 10. Schematic Diagram of Array Models (a) Equilateral Triangular Model, (b) Square Model and (c) Regular Hexagonal Model

We have optimized the weights for three and four and six elements to discover the actual output using the above SNWOM model with initial weights, bias and learning rate also known as coefficient with the appropriate activation function.The radiation pattern in Figure 11 shows the results obtain from conducting SNWOM optimization. The radiation pattern compares the significant of matching between the desired pattern and the optimized pattern. 


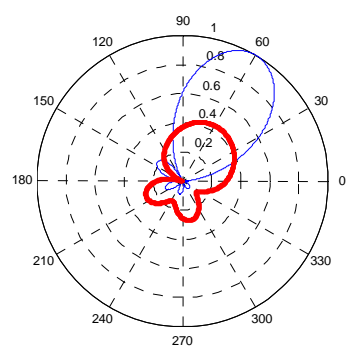

3 Elements

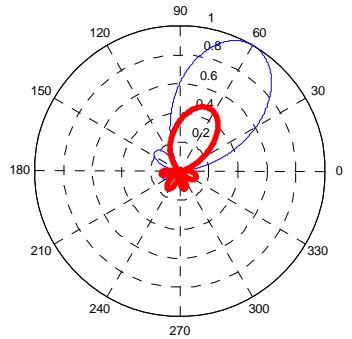

4 Elements

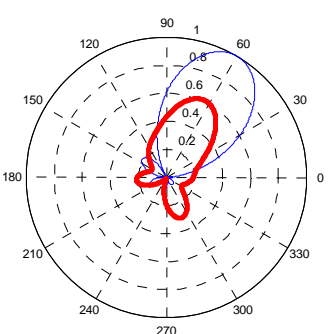

6 Elements

Figure 11. Comparison of Radiation Patterns of 3, 4 and 6 Elements using SNWOM

It can be observed from Figure 11 that as the number of elements increases the optimized beam patterns have better match to the desired beam from three to four to size elements. In addition to beam pattern matching, the beam width is also reduced. A narrow beam would have a greater coverage while utilizing less power as compare to an Omni-directional antenna. However the complex weights optimized from LMS method for the same cases give superior match when the number of elements is increased as shown in Figure 12. In this paper we have compared with LMS method for the formation of a desired beam using antennabased beam forming as opposed to chip-based beam forming. In chip based beam forming multiple beams are created that should constructively add together at the receiver (mobile station), thus requiring the receiver to send signals to the base station transmitter to steer the beam. Where the receiver may have multiple antennas, an ambiguity arises as to the specific antenna that had sent a signal to the transmitter. In the antenna based beam forming, reported herein, the beam former may handle both signal receiver or a single cluster of receivers in one geometrical location, or multiple clusters or antennas as in Figure 5 for instance. The position and velocity of the receiver (MS) antenna may be determined a well-established method [17] where automatic beam steering is needed in the case of mobile receivers. Thus the transmitter antenna beam may be optimized at the MS's present location, rather than the MS's previous location.

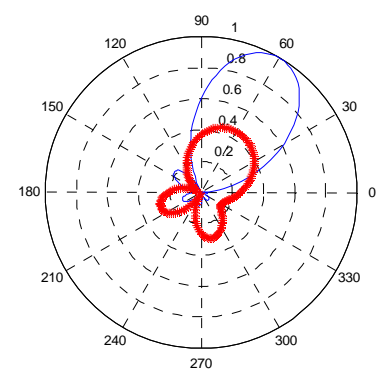

3 Elements

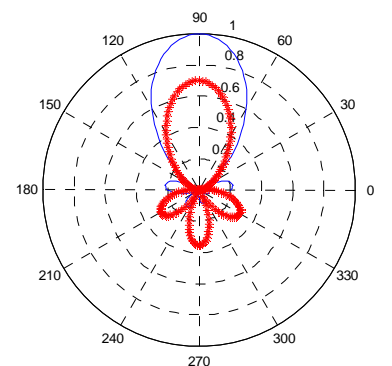

4 Elements

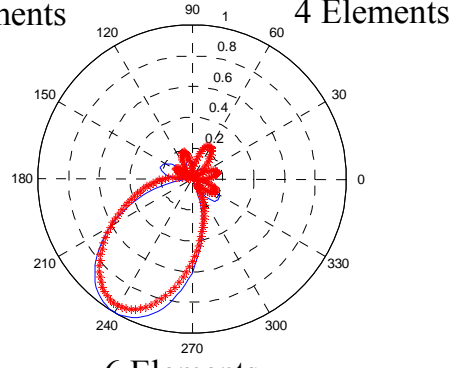

6 Elements

— Desired Beam $\quad * * * * * *$ Optimized Beam

Figure 12. Comparison of Radiation Patterns of 3, 4 and 6 Elements using LMS optimization

\section{CONCLUSIONS}

A simple, accurate and efficient approach to the problem of adaptive beamforming was proposed and implemented using single neuron neural network. The weights were optimized using SNWOM with 
appropriate activation functions and compared with that of traditional LMS method for the comparison of performance. The radiation patterns obtained from optimized weights were close match with the desired radiation patterns when the weights coefficients were real but it was marginally matched for complex weight coefficients. It showed that the activation functions selected for the complex weight coefficients have not fully supported the optimization.

\section{FUTURE WORK}

When the dipoles are placed in straight line along with the selected desired beam, the weight coefficients turned to be real values. For the present setup, the selected nonlinear activation function matched very well and weight coefficients could be optimised easily. However, it will not be the case in real time application where the dipole placement and desired beam function may not be as taken in this model where the expected weight coefficients would be complex values. In order to optimise complex weigh coefficients, the presently proposed nonlinear activation function may not be the most suitable. Therefore, the future work can be focused on selecting appropriate nonlinear activation function in order to optimise complex weights coefficients.

\section{REFERENCES}

[1] H. L. Southall, et al., "Direction finding in phased arrays with a neural network beamformer," IEEE Transactions on Antennas and Propagations, vol. 43, pp. 1369-1374, 1995.

[2] A. H. E. Zooghby, et al., "Performance of radial basis function network for direction of arrival estimation with antenna arrays," IEEE Transactions on Antennas and Propagations, vol. 45, pp. 1611-1617, 1997.

[3] Mozingo and Miller, "Introduction to Adaptive Arrays," New York, Wiley, 1980.

[4] A. H. E. Zooghby, et al., "Neural Network-Based Adaptive Beamforming for One-and Two- Dimensional Antenna Array," IEEE Transactions on Antennas and Propagations, vol. 46, pp. 1891-1893, 1998.

[5] N. Y. Wang, et al., "A new DOA Estimation Technique Based on SubarrayBeamforming," IEEE Transactions on Signal Processing, vol/issue: 54(9), pp. 3279-3290, 2006.

[6] J. L. Fournier, et al., "Phased Array Antenna Controlled by Neural Network FPGA," Loughborough Antennas and Propagation Conference, 14-15 November 2011, Loughborough, UK, pp. 1-5, 2011.

[7] M. Wang, et al., "A neural network approach of Ultra-wideband Near field Adaptive Beamforming," ESANN, Belgium, 2005.

[8] J. R. Mohammed, "Comparative Performance Investigation of Stochastic and Genetic Algorithms under Fast Dynamically Changing Enviornment in Smart Antennas," International Journal of Electrical and Computer Engineering (IJECE), vol/issue: 2(1), pp. 98-105, 2012.

[9] M. Anitha and N. G. Kurahatti, "Neural fuzzy Inference Based Robust Adaptive Beamforming," International Journal of Emerging Technology and Advanced Engineering, vol/issue: 3(11), 2013.

[10] R. H"ansch and O. Hellwich, "Classification of Polarimetric SAR data by Complex Valued Neural Networks," Proceedings of ISPRS workshop, Hannover, Germany, vol. 38, 2009.

[11] M. S. Kim and C. C. Guest, "Modification of Back-propagation for complex-valued-signal processing in frequency domain," International Joint Conference on NeuralNetworks, (San Diego, CA), pp. III-27-III-31, 1990.

[12] T. Kim and T. Adali, "Fully complex multi-layer perception network for non-linear signal processing," Journal of VLCI signal processing, vol. 32, pp. 29-43, 2002.

[13] H. E. Michel, et al., "Artificial Neural Networks using Complex number and Phase encoded weights - Electronic and Optical Implementations," International joint conference on Neural Networks, 2006.

[14] A. Hamid, et al., "New activation functions for complex-valued neural network," International Journal of Physical Sciences, vol/issue: 6(7), pp. 1766-1772, 2011.

[15] P. R. P. Hoole, "Smart Antennas and Signal processing for Communication," Biomedical and Radar Systems, WIT Press, UK, 2001.

[16] S. K. Bodhe, et al., "Beamforming Techniques for Smart Antennas using Rectangular Array Structure," International Journal of Electrical and Computer Engineering (IJECE), vol/issue: 4(2), pp. 257-264, 2014.

[17] X. Wang, et al., "An electromagnetic-time delay method for determining the positions and velocities of mobile stations in a GSM network," J A Kong (Editor), Progress In Electromagnetics Research, vol. 23, pp. 165-186, 1999.

IJECE Vol. 6, No. 5, October 2016: 2300-2309 


\section{BIOGRAPHIES OF AUTHORS}
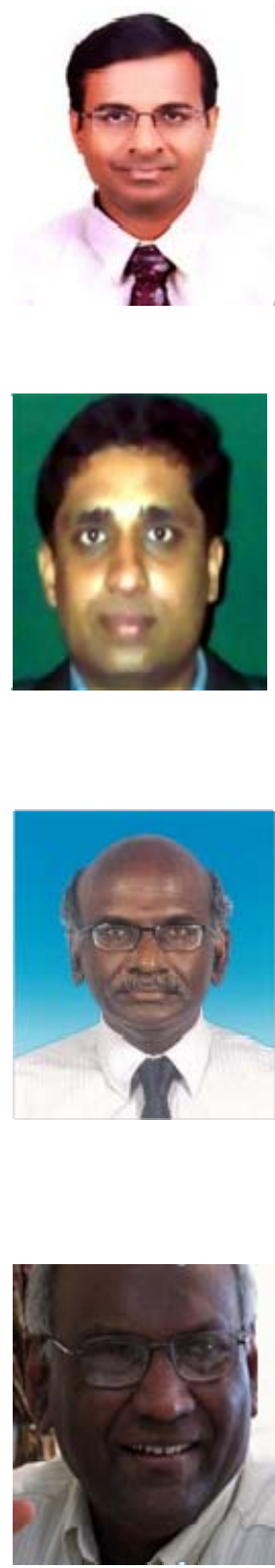

K. S. Senthilkumar received his B.Sc. and M.Sc. Degrees in Computer Science from the University of Peradeniya, Sri Lanka in 1998 and 2003, respectively. Then, he received his M.Tech. Degree in 2006 and Ph.D. Degree in 2011, from Jawaharlal Nehru University, New Delhi, India. He did research on Multiple Robot Terrain exploration and coverage for his Ph.D. Degree in Computer Science. He is a Senior lecturer in the Department of Mathematics and Computer Science, Papua New Guinea University of Technology. His research area covers Robotics, Artificial Intelligence and Soft Computing. He is a Member of the IET.

Dr. K. Pirapaharan, B.Sc. Eng. Hons (Peradeniya, Sri Lanka), M.Eng., PhD (Kinki, Japan), is an Associate Professor of Electrical and Communications Engineering at the University of Technology, Papua New Guinea. Previously he was a Senior Lecturer at Taylor's University in Malaysia. His doctoral research was in microwave and millimeter waves from Kinki University, Japan. From 2001 to 2003, he was a postdoctoral research associate at Centre for Computational Electromagnetics, University of Illinois at Urbana Champaign, USA. From 2004 to 2011, he was a Senior Lecturer in the Electrical and Information Engineering Department at University of Ruhuna, Sri Lanka where he was the head of the department from 2005 to 2008. Dr. Pirapaharan's research interests include wave propagation in inhomogeneous media, adaptive antenna techniques and computational electromagnetics. He is a Member of the IEEE and IET.

Paul R.P. Hoole was born in Jaffna, Sri Lanka in 1958. After having his basic schooling in Jaffna, he earned all his degrees, first degree to postgraduate, in the United Kingdom. He holds an M.Sc degree in Electrical Engineering with a Mark of Distinction from the University of London and an MSc degree in Plasma Science from University of Oxford. His doctorate, the D.Phil. degree, is from the University of Oxford. In his engineering career he has spent time in Singapore, Papua New Guinea, USA, Sri Lanka and Malaysia. After a long career as Professor of Electrical Engineering, because of his interests in lightning engineering, he has just embarked on a job as Professor of Electrical and Electronic Engineering at Universiti Malaysia, Sarawak, Malaysia. Prof. Hoole has authored several papers and books in engineering. His latest book (with K. Pirapaharan and S.R.H. Hoole), Electromagnetics Engineering Handbook, was published by WIT Press, UK, in June 2013.

S. Ratnajeevan H. Hoole, B.Sc. Eng. Hons Cey., M.Sc. with Mark of Distinction London, Ph.D. Carnegie Mellon, is Professor of Electrical and Computer Engineering at Michigan State University in the US. For his accomplishments in electromagnetic product synthesis the University of London awarded him its higher doctorate, the D.Sc. (Eng.) degree, in 1993, and the IEEE elevated him to the grade of Fellow in 1995 with the citation "For contributions to computational methods for design optimization of electrical devices." Prof. Hoole has been Vice Chancellor of University of Jaffna in Sri Lanka, and as Member of the University Grants Commission there, was responsible with six others for the regulation of the administration of all 15 Sri Lankan universities and their academic standards, admissions and funding. Prof. Hoole has been trained in Human Rights Research and Teaching at The René Cassin International Institute of Human Rights, Strasbourg, France, and has pioneered teaching human rights in the engineering curriculum. 